\title{
Field-enlarging transformations and chiral theories
}

\author{
J. Sładkowski ${ }^{\dagger}$ \\ Fakultät für Physik Universität Bielefeld, \\ D-4800 Bielefeld 1, Universitätsstrasse 25, Germany
}

\begin{abstract}
A field-enlarging transformation in the chiral electrodynamics is performed. This introduces an additional gauge symmetry to the model that is unitary and anomaly-free and allows for comparison of different models discussed in the literature. The problem of superfluous degrees of freedom and their influence on quantization is discussed. Several "mysteries" are explained from this point of view.
\end{abstract}

$\dagger$ A. von Humboldt Fellow; permanent address: Dept. of Field Theory and Particle Physics, University of Silesia, Pl 40007 Katowice, Poland. 
Consistent quantization of an anomalous chiral gauge theory have, for a long time, been problematic. In several simple cases, a physically consistent and unitary models can be obtained ${ }^{1-7}$. But it still remains one of the most important open questions in field theory ${ }^{5,8,9}$. To solve the problem one usually adds in a more or less sophisticated way additional terms to the Lagrangian ${ }^{1-7,10-12}$. Another way is to introduce a non-local gauge-fixing or interaction term ${ }^{13-15}$. The resulting theory is then invariant with respect to a restricted gauge symmetry that is not anomalous. Here we would like to apply a field-enlarging transformation to analyse the problem ${ }^{3,16-18}$. This transformation introduces additional scalar degrees of freedom to the system and restores gauge symmetry, although not always the one one started with. It is then possible to show explicitely the relations among various proposals and how the mechanism works. The conventional common part of Lagrangian for the discussed in the literature models is (chiral electrodynamics):

$$
L=-\frac{1}{4} F^{\mu \nu} F_{\mu \nu}+\bar{\psi}\left[i \partial^{\mu} \gamma_{\mu}-\frac{e}{2}\left(1+\gamma^{5}\right) A_{\mu} \gamma^{\mu}\right] \psi .
$$

This Lagrangian is invariant with respect to

$$
\delta A_{\mu}=\partial_{\mu} \alpha
$$




$$
\begin{aligned}
& \delta \psi=-i \alpha \frac{e}{2}\left(1-\gamma^{5}\right) \psi \\
& \delta \bar{\psi}=i \frac{\alpha e}{2} \bar{\psi}\left(1+\gamma^{5}\right),
\end{aligned}
$$

where $\alpha$ is an arbitrary real function. Unfortunately, this gauge invariance is spoiled at the quantum level ${ }^{19}$. Let us perform the following field-enlarging transformation ${ }^{3,16-18}$

$$
A_{\mu} \rightarrow A_{\mu}-\partial_{\mu} \phi \equiv g_{\mu}(A, \phi)
$$

in the Lagrangian (1). The transformed Lagrangian has the form

$$
L=-\frac{1}{4} F^{\mu \nu} F_{\mu \nu}+\bar{\psi}\left[i \partial^{\mu} \gamma_{\mu}-\frac{e}{2}\left(1+\gamma^{5}\right)\left(A_{\mu} \gamma^{\mu}-\gamma^{\mu} \partial_{\mu} \phi\right)\right] \psi
$$

Although this seems to be trivial at the first sight, especially, when the gauge field mass term and/or gauge-fixing term for the symmetry (2) are absent, the consequences are not ${ }^{3,16-18}$. The reason is that that quantization of a chiral fermion results in a non-trivial interaction that breaks the classical gauge symmetry (anomaly). It is also possible to redefine the fermion field via

$$
\psi \rightarrow e^{f\left(\phi, \gamma^{5}\right)} \psi
$$




$$
\bar{\psi} \rightarrow e^{f^{\dagger}\left(\phi, \gamma^{5}\right)} \bar{\psi}
$$

Then the fermion field is not invariant with respect to (5). In fact, it is also possible to choose the function $f$ so that the scalar field $\phi$ is absent from the Lagrangian (4). But then one should worry about the Jacobian in the fermionic sector. We have chosen the simplest field redefinition so that everything is explicit! The transformation (6) introduces the following additional Abelian gauge symmetry to the theory ${ }^{3,16-18}$ :

$$
\begin{gathered}
\delta \phi(x)=\bar{\alpha}(x) \\
\delta A_{\mu}(x)=-\int d^{n} x d^{n} y\left(\frac{\delta g^{\mu}(A, \phi)}{\delta A_{\nu}}\right)^{-1}(x, y) \frac{\delta g_{n u}(A, \phi)}{\delta \phi} \bar{\alpha}(z)=\partial_{\mu} \bar{\alpha}(x) \\
\delta \psi=\bar{\psi}=0
\end{gathered}
$$

where $\bar{\alpha}$ is an arbitrary real function. To quantize this model we have to fix both gauge symmetries ${ }^{20}$.

Now, we are prepared to analyse the problem of quantization of an anomalous chiral gauge theory. The authors of Ref. 13 and 14 proposed to perform the non-local transformation 


$$
A_{\mu} \rightarrow A_{\mu}^{g}=A_{\mu}-\partial_{\mu} \frac{1}{\square} \partial_{\nu} A^{\nu}
$$

in (1). The resulting theory

$$
L=-\frac{1}{4} F^{\mu \nu} F_{\mu \nu}+\bar{\psi}\left[i \partial^{\mu} \gamma_{\mu}-\frac{e}{2}\left(1+\gamma^{5}\right) A_{\mu}^{g} \gamma^{\mu}\right] \psi
$$

is then invariant with respect to

$$
\begin{gathered}
\delta A_{\mu}=\partial_{\mu} \alpha \\
\delta \psi=\delta \bar{\psi}=\delta A_{\mu}^{g}=0 .
\end{gathered}
$$

This symmetry is anomaly-free because the fermion field transforms in a trivial way ${ }^{21}$. It can be shown that such non-local theories are unitary and consistent ${ }^{13-15}$. Unfortunately, these conclusions usually concern the additional gauge symmetry that has been introduced to the theory in question, but not the one we started with. The discussed above model is still anomalous with respect to the original $U(1)$ gauge symmetry. Such a Lagrangian might yield a physically acceptable theory, but this is far from being a rule ${ }^{1}$. We should get rid of the anomalous symmetry. The simplest solution is the following. Let us try to quantize the model given by Eq.4. First, let us break the original gauge symmetry (2) by the non-local gauge fixing condition 


$$
\phi-\frac{1}{\square} \partial_{\mu} A^{\mu}=0
$$

The Lagrangian has the form (we omit the Faddeev-Popov ghost term)

$$
L=-\frac{1}{4} F^{\mu \nu} F_{\mu \nu}+\bar{\psi}\left[i \partial^{\mu} \gamma_{\mu}-\frac{e}{2}\left(1+\gamma^{5}\right)\left(A_{\mu} \gamma^{\mu}-\gamma^{\mu} \partial_{\mu} \phi\right)\right] \psi+\rho\left(\phi-\frac{1}{\square} \partial_{\mu} A^{\mu}\right)
$$

where an auxiliary scalar field $\rho$ has been introduced to exponentiate the functional Dirac $\delta$-function that force the gauge condition (9). We can perform the path integral over the scalar fields. This results in

$$
L=-\frac{1}{4} F^{\mu \nu} F_{\mu \nu}+\bar{\psi}\left[i \partial^{\mu} \gamma_{\mu}-\frac{e}{2}\left(1+\gamma^{5}\right)\left(A_{\mu} \gamma^{\mu}-\partial_{\nu} \gamma^{\nu}\left(\frac{1}{\square} \partial_{\mu} A^{\mu}\right)\right)\right] \psi .
$$

This is the Lagrangian given by (7) ${ }^{13}$ with the $A^{g}$ field written explicitly! The additional gauge symmetry (8) is the same as (5). Of course, other gauge conditions lead to different representation of the model. This shows that the proposal put forward in Refs 13 and 14 is to break the original symmetry (2) and to introduce a new one that is anomaly-free (and in some sense trivial because it leaves fermions invariant). In fact, it can be shown that the transformation (6) chooses the covariant gauge

$$
\partial_{\mu} A^{\mu}=0 .
$$


So we should not speak of a transformation but rather of a gauge fixing condition. More sophisticated gauge conditions breaking (5) would result in more complicated Lagrangians.

Jackiw and Rajaraman, in their seminal paper ${ }^{1}$, discovered that the two dimensional chiral Schwinger model yields a consistent and unitary, although anomalous and not gauge-invariant, theory. After this, several other consistent anomalous models have been put forward. They have the following general form

$$
\begin{gathered}
L=-\frac{1}{4} F^{\mu \nu} F_{\mu \nu}+\bar{\psi}\left[i \partial^{\mu} \gamma_{\mu}-\frac{e}{2}\left(1+\gamma^{5}\right) A_{\mu} \gamma^{\mu}\right] \psi \\
+\frac{1}{2} B^{2}-B \partial_{\mu} A^{\mu}+\partial_{\mu} \bar{c} \partial^{\mu} c+m^{2} K(\phi, A)+\phi P(A),
\end{gathered}
$$

where B , c and P denote the auxiliary field that linearize the gauge condition, the appropriate ghosts and the Pontryagin term ${ }^{22}$, respectively. Several forms of the K-term have been discussed in the literature ${ }^{3,1-7,10-13}$. In the $1+1$ dimensional case, it is possible to calculate the functional integral over the fermions ${ }^{22}$ in (1). Then one can apply the transformation (3) ${ }^{3}$. This leads (after "reintroduction" of fermions and addition of the gauge fixing and ghost terms) to

$$
m^{2}=\frac{e^{2}}{4 \pi}(a-1)
$$


where $a$ is the quantization (regularization) ambiguity parameter [1] and

$$
K(\phi, A)=\frac{1}{2} \partial_{\mu} \phi \partial^{\mu} \phi-\partial_{\mu} \phi A^{\mu} .
$$

This form correspond to a theory that posses the additional gauge symmetry (5). This additional symmetry is the unexpected gauge invariance discovered in Ref. 12 after adding the Wess-Zumino term to the chiral electrodynamics Lagrangian. This form of the K-term has been recognized in Ref. 14 as the one corresponding to the model discussed by Jackiw and Rajaraman ${ }^{1}$. This not so, because the additional symmetry is absent in their model ${ }^{3}$. One has to break the additional symmetry in order to get the Jackiw and Rajaraman model ${ }^{3}$. Faddeev and Shatashvili ${ }^{10}$ have chosen $K=0$. This corresponds to the $a=1$ case of Eq. (14a). Path integration over the scalar field $\phi$ leads to the condition $P=0$ that ensure the invariance with respect to (5). The form proposed by Rajeev ${ }^{11}$

$$
K(\phi, A)=\frac{1}{2}\left(\partial_{\mu} \phi-A_{\mu}\right)\left(\partial^{\mu} \phi-A^{\mu}\right),
$$

has an additional term $\frac{1}{2} e^{2} A_{\mu} A^{\mu}$ that breaks the the symmetry (8). It should be interpreted as a mass term for the gauge boson (Stückelbeg formalism ${ }^{23}$ ). Finally, Thompson and Zhang proposed to take

$$
K(\phi, A)=\partial_{\mu} \rho\left(\partial^{\mu} \phi-A^{\mu}\right)
$$


where $\rho$ is an auxiliary scalar field. This model is equivalent to the ordinary chiral Schwinger model ${ }^{1,3}$. This can be seen by integrating over the scalar fields. Note that this differs from (4) ${ }^{13}$ in that the additional symmetry is broken. This shows once more that for any value of a the original gauge symmetry is lost in the quantization process ${ }^{1,22}$. The above analysis shows that the consistency of quantization of the discussed models has common roots that have been discovered by Jackiw and Rajaraman ${ }^{1}$ because the differences in the K-terms can be regarded as different gauge fixing terms for the symmetry (5). Note that the discussed Lagrangians can be obtained also by more sophisticated ways ${ }^{4-7,24}$. The important fact is that the additional symmetry (5) reveals itself in every case, although it might not be obvious, e.g. in the field-antifield formalism it is fixed in the due process ${ }^{5,23-25}$.

The important question can a field-enlarging transformation help to construct a non-trivial anomaly-free theory? arises. The answer may be affirmative. It has been observed that a theory can posses a BRST symmetry ${ }^{26}$ that is not a symmetry of the Lagrangian but only of the functional integral. This means that several symmetries, if "broken correctly", may result in a anomaly-free subsymmetry (cancellation of the anomalous terms in the fermionic determinant). To shed more light on the problem, let us consider the BRST symmetries that correspond to (2) and (5). The general formula 
for a BRST current associated to the fields that appear in (13) is ${ }^{14,20,26}$

$$
J_{B R S T}^{\mu}=F^{\mu \nu} \partial_{\nu} c-\phi \frac{\partial P}{\partial \partial_{\mu} A_{\nu}} \partial_{\nu} c+e J_{L}^{\mu} c+B \partial^{\mu} c-\frac{\partial K}{\partial \partial_{\mu} \phi} \delta_{B R S T} \phi
$$

where $J_{L}$ denotes the left fermion current. Its divergence is

$$
\partial J_{B R S T}=e \partial J_{L} c-P \delta_{B R S T} \phi-\left(\frac{\partial K}{\partial \partial A_{\mu}} \partial_{\mu} c+\frac{\partial K}{\partial \partial_{\mu} \phi} \partial_{\mu} \delta_{B R S T} \phi\right),
$$

so that if $\mathrm{K}$ is gauge invariant and $\delta_{B R S T} \phi=c, J_{B R S T}^{\mu}$ is conserved $\left(\partial J_{B R S T}=\right.$ 0) ${ }^{13}$. It is obvious that this condition is fulfilled by the K-terms given by (15) and (16). This conserved BRST charge corresponds to the diagonal part of (2) and (5) $(\alpha=\bar{\alpha})$. The form of the K-term given by (14) (and its special case $K=0$ ) defines a model that is gauge invariant with respect to (5) and the appropriate BRST current is also conserved. Unfortunately, the described above phenomenon seems to require additional fields or/and non-local terms. It is also obscure if, and to what extent, it can work in more than $1+1$ dimensional spacetime. The Batalin-Vilkovisky or the discussed here field-enlarging (Stückelberg ${ }^{23}$ ) formalism should be helpful in analysing this problem. Especially, the role of the additional symmetry should be explored. This problem is under investigation. Recently, similar ideas has been discussed in the context of the $W_{2}-$ gravity $^{7}$. 
Acknowledgements. The author would like to thank prof. R. Kögerler and dr K. Kołodziej for stimulating and helpful discussions, and the anonymous Referee for helpful remarks and bringing attention to the references $4-7$. This work has been supported in part by the Alexander von Humboldt Foundation and the Polish Committee for Scientific Research under the contract KBN-PB 2253/2/91. 


\section{References}

[1] R. Jackiw and R. Rajaraman, Phys. Rev. Lett. 54, 1219 (1985).

[2] P. Mitra, Phys. Lett. B284, 23 (1992).

[3] J. Sładkowski, Phys. Lett. B296, 361 (1992).

[4] O. Babelon F. A. Schaposnik and C. M. Viallet, Phys. Lett. B177, 385 (1986).

[5] J. Gomis and J. Paris, Nucl. Phys B395, 288 (1993).

[6] N. R. F. Braga and L. Montani, Phys. Lett. B264, 125 (1991).

[7] F. De Jonghe, R. Siebelink and W. Troost, Phys Lett B 306, 295 (1993).

[8] M. Abdelhafiz, K. Kołodziej and M. Zrałek, Jagellonian Univ. preprint, TPJU-2/86 ,1986 (unpublished).

[9] J. Sładkowski and M. Zrałek, Phys. Rev. D45, 1701 (1992).

[10] L. D. Faddeev and S. L. Shatashvili, Phys. Lett. B167, 225 (1986).

[11] S. G. Rajeev, MIT Report No CTP 1405 (1986).

[12] K. Harada and I. Tsutsui, Phys. Lett. B183, 311 (1987). 
[13] G. Thompson and R. Zhang, Mod. Phys. Lett. A2, 869 (1987).

[14] A. Della Serva, L. Maspieri and G Thompson, Phys. Rev. D37, 2347 (1998).

[15] G. L. Demarco, C. Fosco and R. C. Trinchero, Int. J. Mod. Phys. A7, 5459 (1992).

[16] J. Alfaro and P. H. Damgaard, Ann. Phys. (NY) 202, 398 (1990).

[17] J. Alfaro and P. H. Damgaard, CERN-preprint CERN-TH6455/92 (1992).

[18] A. Hosoya and K. Kikkawa, Nucl. Phys. B101, 271 (1975).

[19] J. Bell and R. Jackiw, Nuovo Cim. 60A, 47 (1969); S. Adler, Phys. Rev. 177, 2426 (1969).

[20] T. Kugo and I. Ojima, Progr. Theor. Phys. Suppl. 66, 1 (1979).

[21] K. Fujikawa, Phys. Rev. D21, 2848 (1980).

[22] R. Jackiw, in Relativity, Groups and Topology II, edited by B. DeWit and R. Stora (North-Holland, Amsterdam, 1984), p. 224.

[23] J. Sładkowski, submitted to Zeitschr. Phys. C. 
[24] J. Alfaro and P. H. Damgaard, CERN-preprint CERN-TH6788/93 (1993); to apear in Nucl. Phys. B.

[25] T. Fujiwara, Y. Igarashi and J. Kubo, Nucl. Phys. B341, 695 (1990).

[26] C. Becci, A. Rouet, and R. Stora, Phys. Lett. B52, 344 (1974);

I. V. Tyutin, Lebedev Report No. FIAN 39 (1975). 